\title{
Recuperação de portadora FM em freqüências de microondas utilizando malha realimentada e filtro SAW
}

\author{
Marbey M. Mosso, Vanessa P. R Magri, Rodolfo A. A Lima e Claumir S. Silva
}

\begin{abstract}
Resumo-Este trabalho apresenta um circuito ativo para a recuperação de portadoras de $R F$ na faixa de microondas. $O$ circuito consiste em uma malha de realimentação construída sobre o substrato FR-4, utilizando somente componentes SMD (Surface Mounted Device) comerciais, amplificação balanceada em quadratura e tendo como elemento ressonante um filtro SAW (Surface Acustic Wave). A malha básica de oscilação foi simulada e resultados experimentais demonstram a recuperação da portadora de um sinal modulado FM em 1,25 GHz.
\end{abstract}

Palavras-chave - Osciladores de microondas, amplificadores realimentados, recuperação de portadora.

Abstract- An active circuit for carrier recovery in the microwave range is presented. The circuit consists of a feedback loop, fabricated on an FR-4 substrate, using only commercial SMD components, quadrature-balanced amplification and having a surface acoustic waver (SAW) filter as the resonant element. The basic oscillator loop was simulated and experimental results demonstrate the carrier recovery of an FM modulated signal at 1.25 GHz.

Keywords - Microwave oscillator, feedback amplifier, carrier recovery.

\section{INTRODUÇÃO}

Amplificadores realimentados (Feedback Amplifiers) têm sido intensamente utilizados em circuitos de microondas [1][4]. Para estes dispositivos, a malha de realimentação utiliza componentes ativos e passivos que atendem a condição de oscilação básica e permitem a monitoração do sinal.

A utilização desse tipo de malha como osciladores ILO (Injection-Locked Oscillators) [5] para a recuperação de portadora tem se mostrado mais eficiente do que malhas tradicionais PLL (Phase-Locked Loop). Uma malha ILO apresenta menor probabilidade de perda de sincronização, pois sua resposta é mais rápida que a das malhas PLL [6].

Foi selecionado um conjunto de componentes comerciais de microondas do tipo SMD e uma configuração inicial de um oscilador ILO foi simulada na ferramenta computacional Agilent $^{\mathrm{TM}}$ ADS (Advanced Design System) para operar na freqüência de 1,25 GHz. Desta forma, a configuração final pôde ser montada em uma placa de circuito impresso.

Marbey M. Mosso, Rodolfo A. A. Lima e Vanessa P. R. Magri, CETUC Centro de Estudos em telecomunicações, PUC-Rio, RJ, Brasil, E-mails: marbey@cetuc.puc-rio.br, rlima@cetuc.puc-rio.br, vanessamagri@cetuc.pucrio.br. Claumir S. Silva, Grupo de Guerra Eletrônica - IPqM Instituto de Pesquisas da Marinha, RJ, Brasil, claumir@cetuc.puc-rio.br.
Um conjunto de testes experimentais utilizando um analisador de redes, um analisador de espectro e um osciloscópio digital, permitiu que o circuito fosse convenientemente ajustado para a condição de recuperação de portadora. O circuito foi ajustado para operar próximo à condição de oscilação [1], isto é, ganho de malha ligeiramente inferior à unidade e fase próxima de $2 \cdot n \cdot \pi$, para $n$ inteiro. Um sinal modulado em FM foi introduzido na entrada do circuito e a portadora associada foi recuperada em sua saída.

O módulo realizado em circuito impresso (CI) é descrito na seção II. Na seção III é apresentada a simulação da malha de oscilação no aplicativo Agilent ${ }^{\mathrm{TM}}$ ADS. Na seção IV são apresentados os resultados experimentais obtidos para o circuito desenvolvido. Na seção $\mathrm{V}$ são analisados os resultados, visando à discussão da validade e aplicabilidade do procedimento de projeto proposto.

\section{PROJETO DO CIRCUITO}

Nesta seção é descrita a configuração de um oscilador de microondas baseado em uma topologia de amplificador realimentado (Feedback Amplifier), cuja malha de realimentação é composta por componentes passivos. A configuração do oscilador apresentado pode funcionar como ILO para recuperação de portadora.

A Fig. 1 apresenta o diagrama em blocos do circuito para a realização do módulo protótipo. O circuito foi projetado para operação em 1,25 GHz.

Como elemento de ganho, foi utilizado um par casado de amplificadores de potência (Minicircuits Mera556 Dual Matched MMIC Amplifiers), com tipicamente $20 \mathrm{~dB}$ de ganho e $+18 \mathrm{dBm}$ de potência de saída, na faixa $\mathrm{DC} \sim 2,2 \mathrm{GHz}$. Foram utilizadas duas híbridas de $90^{\circ}$ para criar uma estrutura de amplificação balanceada em quadratura [7].

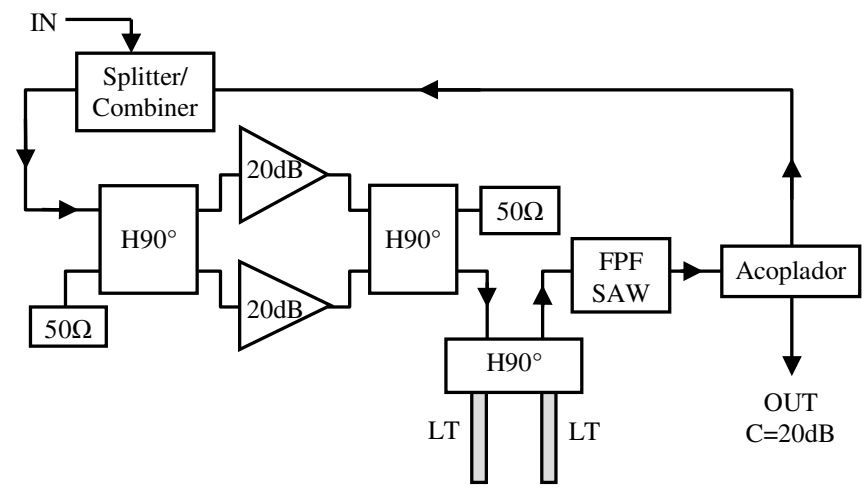

Fig. 1. Diagrama em bloco do circuito proposto para recuperação de portadora através da configuração de malha amplificada realimentada. 
O elemento ressonante da malha é um filtro de ondas acústicas SAW (Surface Acoustic Wave filter) [8], que fornece um alto fator de qualidade realimentado na frequiência de oscilação do circuito. A escolha do filtro SAW é fundamental para o projeto, pois vai definir a frequiência e a faixa de operação do circuito. Foi utilizado um filtro comercial da NJR Corporation (NSVA254) em 1,25 GHz.

O conjunto formado pela terceira hibrida $90^{\circ}$ com os trechos de linhas de transmissão (LT) de comprimento ajustável permitem que a fase da malha de realimentação atinja o valor $2 \cdot n \cdot \pi$, garantindo assim a condição de desvio de fase total igual a zero para a condição de oscilação na frequiência desejada [1].

A injeção de sinal é realizada através de um divisor/somador, enquanto a saída do circuito se dá por meio de um acoplador direcional; foi utilizado um elemento comercial com fator de acoplamento $20 \mathrm{~dB}$.

O projeto foi realizado em uma placa de circuito impresso sobre substrato FR4 $\left(\varepsilon_{r}=4,5\right)$. Foram utilizados componentes comerciais SMD (Surface Mount Device), selecionados no catálogo da MiniCircuits ${ }^{\mathrm{TM}}$ : Hibridas de $90(\mathrm{QCN} 19 ; 1,1 \mathrm{GHz}$ $\sim$ 1,925 GHz), divisor/ combinador (SBB2-13; $950 \mathrm{MHz} \sim 1,3$ $\mathrm{GHz}$ ) e acoplador (JDC20-5; $50 \mathrm{MHz} \sim 1,5 \mathrm{MHz}$ ).

\section{SimulaÇÃO DA MALHA DE OSCILAÇÃO}

Utilizando o aplicativo Agilent ${ }^{\mathrm{TM}}$ ADS, o circuito proposto com os componentes selecionados foi simulado. A Fig. 2 apresenta o diagrama de blocos da simulação do circuito na configuração de Balanço Harmônico no ADS. Na Fig.3, é apresentado o resultado da simulação para a malha, tendo sido observada oscilação em $1,25 \mathrm{GHz}$ e potência de saída de aproximadamente $0,5 \mathrm{dBm}$.

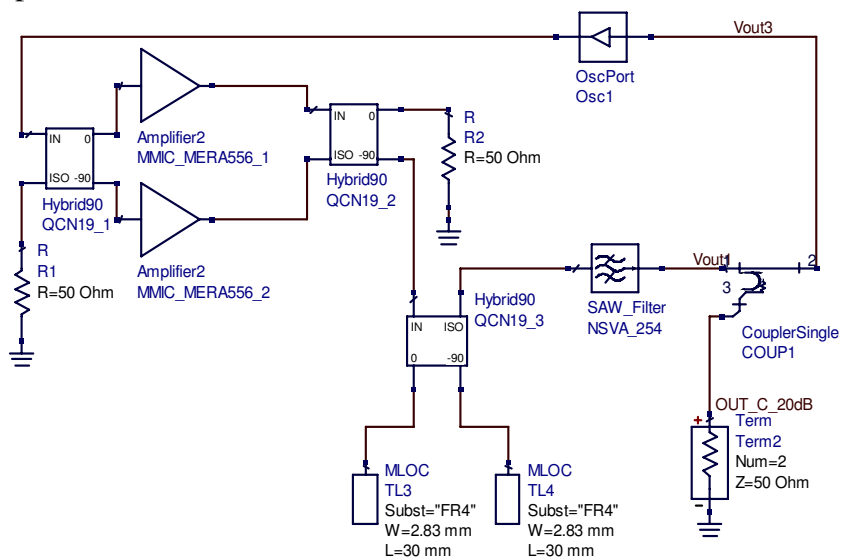

Fig. 2. Diagrama de blocos da simulação de Balanço Harmônico no aplicativo Agilent ${ }^{\mathrm{TM}} \mathrm{ADS}$, para a condição de oscilação do módulo projetado.

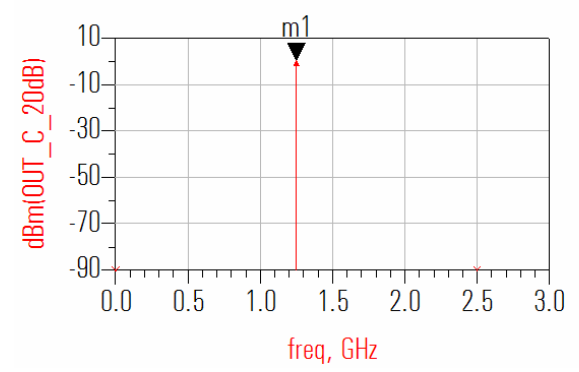

Fig. 3. Resultado da simulação para o oscilador em 1,25 GHz.

\section{MEDIÇÕES E RESULTADOS PRÁTICOS.}

Objetivando o teste do módulo desenvolvido, no laboratório do CETUC, como recuperador de portadora, foi construído um protótipo do circuito projetado, de acordo com o diagrama da Fig. 1 e utilizando os componentes descritos na seção II. A topologia do circuito, na configuração microstrip sobre substrato FR-4, foi desenvolvida de acordo com as máscaras de solda dos componentes, utilizando linhas de $50 \Omega$. A Fig. 4 apresenta uma foto do circuito montado no CETUC.

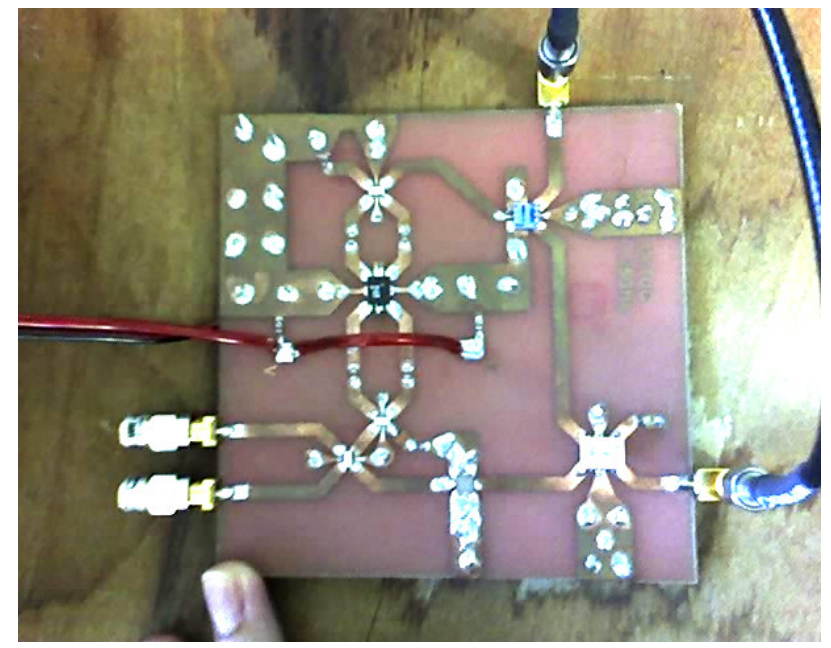

Fig. 4. Foto do módulo recuperado de portadora montado no CETUC.

O circuito foi analisado como amplificador realimentado em um analisador de redes escalar HP8757A e sua resposta em freqüência é apresentada na Fig. 5 para o intervalo de 0,9 a 2,0 GHz. Observando o circuito da Fig 1, a resposta da Fig. 5 corresponde à saída obtida na porta OUT (porta 2) quando é aplicado na porta IN (porta 1) um sinal de entrada RF, com potência $0 \mathrm{dBm}$. Desta forma, obteve-se na saída do acoplador um sinal em $1,25 \mathrm{GHz}$ com perda de inserção de $-12.87 \mathrm{~dB}$ e alto fator de qualidade. Descontando o fator de acoplamento de $20 \mathrm{~dB}$ do acoplador direcional na saída, o ganho obtido pelo módulo foi de $7,13 \mathrm{~dB}$ em $1,25 \mathrm{GHz}$ para essas condições.

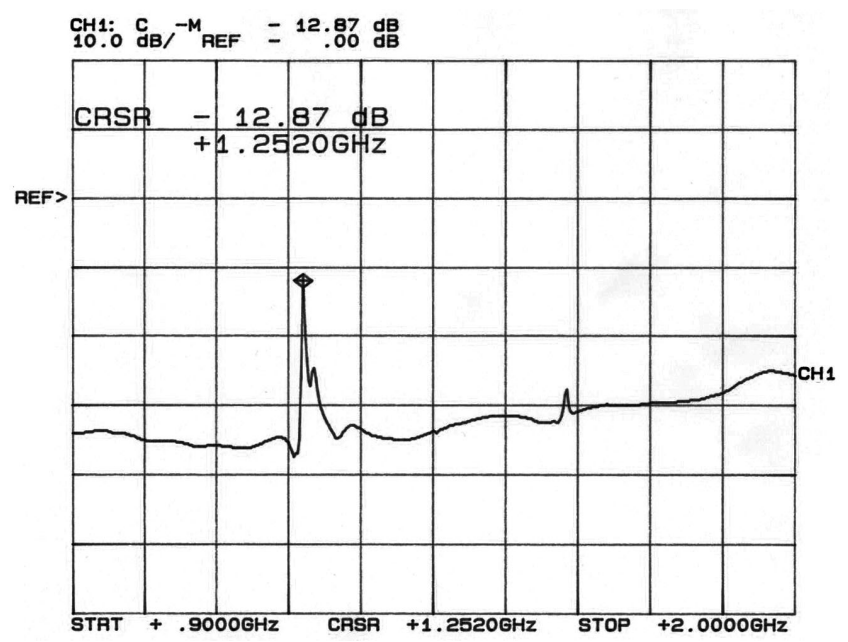

Fig. 5. Resposta em freqüência $\left(\left|S_{21}\right|\right)$ do módulo projetado, observada em um analisador de redes escalar. 
Em seguida, o módulo foi utilizado para a recuperação da portadora de um sinal modulado FM. O setup montado para teste é apresentado no diagrama da Fig. 6. Um gerador de varredura sintetizado HP83752B é utilizado para fornecer uma portadora em 1,255 GHz modulada em FM. Para isso, utilizou-se em sua entrada FM um gerador de funções senoidal em $500 \mathrm{kHz}$ com tensão $0,44 \mathrm{~V}_{\mathrm{p}-\mathrm{p}}$.

O sinal modulado FM obtido, representado por (1) na Fig. 6, foi injetado na malha do recuperador, o módulo ILO. A saída da malha está representada como (2) na Fig. 6, e corresponde à portadora recuperada.

Os sinais (1) e (2) foram registrados em um analisador de espectro elétrico Anritsu MS2661C e são comparados na Fig. 7. Pode-se observar que o sinal modulado FM (1), representado pela linha tracejada na Fig. 7, tem uma largura de banda de aproximadamente $10 \mathrm{MHz}$, na qual não se distingue a portadora. A curva cheia na Fig. 7 corresponde à saída da malha de recuperação, podendo-se observar claramente a portadora recuperada (2).

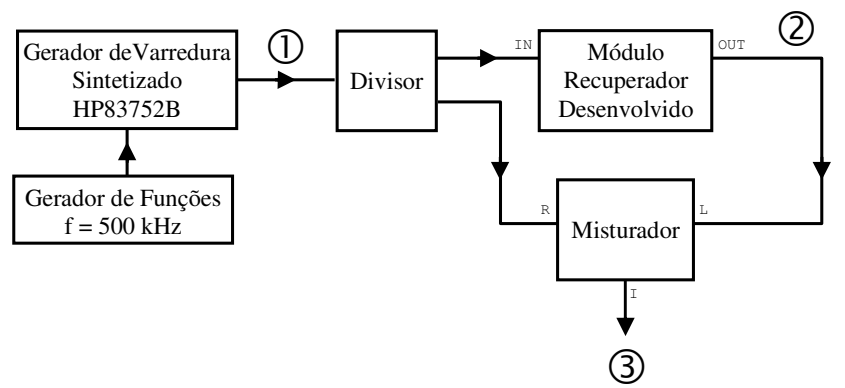

Fig. 6. Esquema do setup para demonstração da recuperação de portadora por meio da análise do espectro do sinal: (1) sinal modulado FM; (2) portadora recuperada; e (3) sinal modulado rebatido para banda básica utilizando a portadora recuperada.

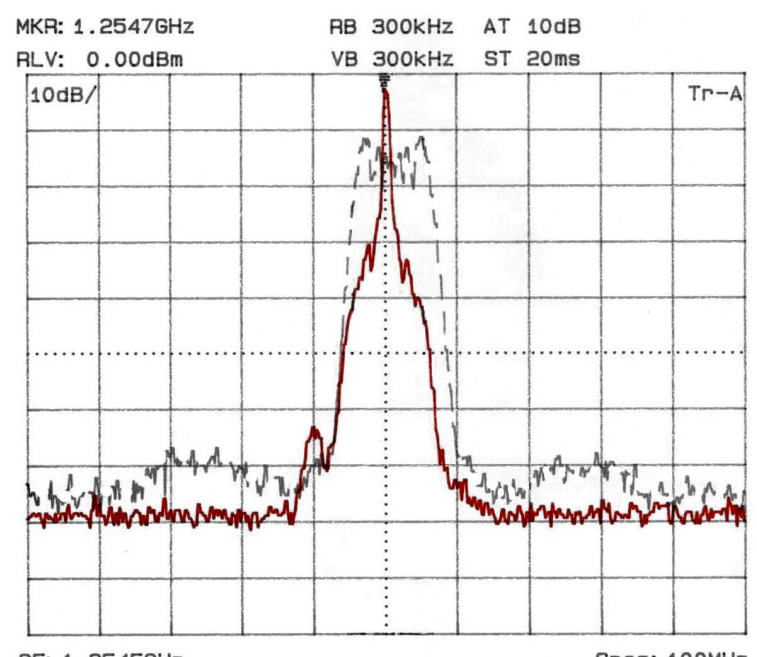

$\mathrm{CF}: 1.2545 \mathrm{GHz}$

Span: $100 \mathrm{MHz}$

Fig. 7. Espectro do (1) sinal modulado FM com $10 \mathrm{MHz}$ de largura de banda (linha tracejada); e (2) portadora recuperada em $1,2547 \mathrm{GHz}$ (linha cheia).

Voltando ao setup da Fig. 6, observa-se que o sinal modulado em FM oriundo de (1) e extraído por um divisor está excitando a porta "sinal" (R) de um misturador. O mesmo divisor fornece o sinal modulado ao recuperador de portadora.
A portadora recuperada (2) excita a porta "oscilador local" (L) do mesmo misturador. Este procedimento translada o sinal modulado para a banda básica. A Fig. 8 apresenta em detalhe o espectro do sinal modulado FM (1). Na Fig. 9, encontra-se o espectro do sinal modulado rebatido para banda básica (3) $\mathrm{Na}$ Fig. 8, o sinal foi atenuado $16 \mathrm{~dB}$ para melhor comparação visual com o resultado da Fig. 9, uma vez que a escala vertical do analisador de espectro é digital e não permite ajuste fino. $\mathrm{O}$ resultado obtido na Fig. 9 incorpora o ruído de fase do oscilador local do analisador de espectro.

Comparando as Figs. 8 e 9, observa-se semelhança satisfatória entre os espectros de sinal modulado e rebatido medidos. Este resultado indica que o sinal de saída da malha de recuperação é coerente com a portadora modulada, pois o aspecto do sinal modulado se manteve após o rebatimento, apresentando também estabilidade no tempo.

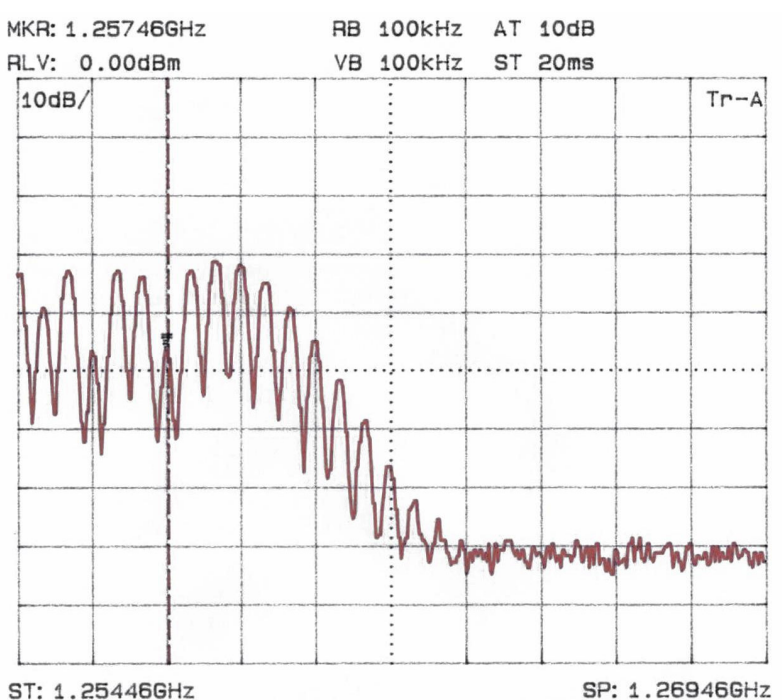

Fig. 8. Espectro do sinal modulado FM em 1.255 GHz.

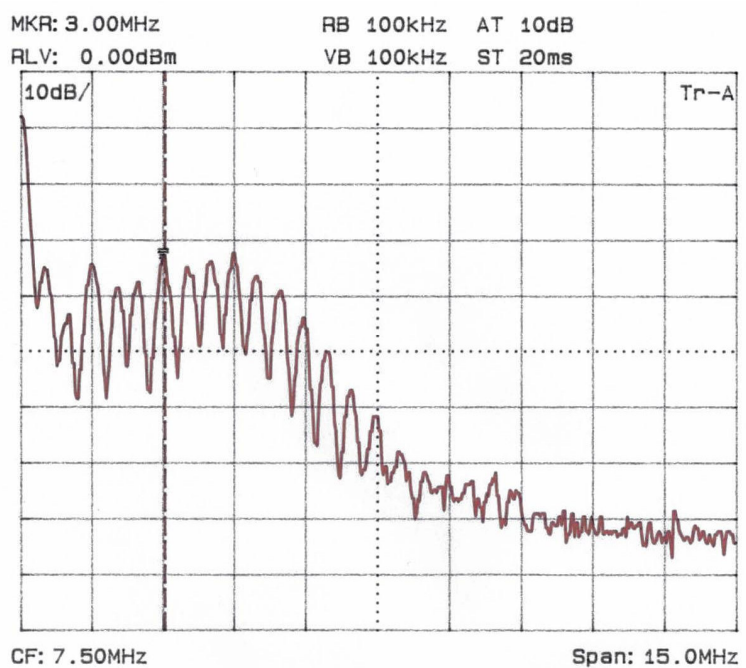

Fig. 9. Espectro do sinal modulado rebatido para banda básica pela portadora recuperada. 


\section{COMENTÁRIOS FINAIS E CONCLUSÕES.}

De acordo com os resultados obtidos, o módulo projetado, apresenta alto fator de qualidade, mesmo com a utilização de componentes SMD em um circuito impresso sobre substrato FR-4 comercial. Esse resultado é obtido devido ao efeito de realimentação da malha. O módulo é capaz de operar como recuperador de portadora em $1,25 \mathrm{GHz}$ para sinais de modulação FM, em uma configuração de amplificador realimentado em malha.

O mesmo módulo utilizado neste trabalho, com ajustes no modo de operação, foi utilizado no laboratório do CETUC para recuperação de portadora modulada FM em diferentes condições de modulação, e pode ser utilizado com outros tipos de modulação, tais como BPSK, QAM etc.

O modelo projetado pode ser adaptado para outras faixas de operação em frequiências de microondas, desde que os componentes SMD utilizados, em especial as hibridas de $90^{\circ} \mathrm{e}$ o filtro SAW, sejam devidamente selecionados entre componentes disponíveis para a frequiência desejada. Entretanto, não foram encontrados filtros SAW para freqüências acima de $2 \mathrm{GHz}$, por limitações da tecnologia. Outras soluções para elemento ressoador de alto Q podem ser adotadas para frequiências superiores.

O módulo apresentado poderá ser futuramente realizado através de um MMIC de silício, utilizando configurações branch-line como híbridas de $90^{\circ}$ para aplicações em freqüências utilizadas em sistemas wireless Wi-Fi e WiMAX.

\section{AGRADECIMENTOS}

Os autores gostariam de agradecer à Agilent $^{\mathrm{TM}}$ e à NJR Corporation, pelo apoio e colaboração.

\section{REFERÊNCIAS}

[1] B. Nikolic, M. Slavoljub, "A General method of feedback amplifier analysis". In: 1998 IEEE International Symposium on Circuits and Systems, ISCAS'98, 1998, Monterey. Proceedings... California, USA: IEEE, 1998, vol. 3, pp. 415-418.

[2] I. Koudar, "Variable gain differential current feedback amplifier". In: 2004 IEEE Custom Integrated Circuits Conference, 2004. Proceedings... IEEE, 2004, pp. 659-662.

[3] E. M. Cherry, "Feedback amplifier configurations", IEE Proceedings Circuits, Devices and Systems, vol. 147, no. 6, pp. 334-346, Dec. 2000.

[4] B. P. Santos, "Otimização do ruído de fase de osciladores na faixa de microondas". Dissertação de Mestrado. Rio de Janeiro, PUC-Rio, Departamento de Engenharia Elétrica, 2005.

[5] R Adler, "A study of locking phenomena in oscillators", Proceedings of the IEEE, vol. 61, no. 10, pp. 1380-1385, Out. 1973.

[6] P. J. Edmonson, P. M. Smith, C. K. Campbell, "Injection Locking Techniques for a 1-GHz Digital Receiver Using Acoustic-Wave Devices", IEEE Transactions on Ultrasonics, Ferroelectrics and Frequency Control, vol. 39, no. 5, pp. 631-637Set. 1992.

[7] A. Podcameni, C. R. Riback, "An Amplifier Linearization Method Based on a Quadrature Balanced Structure". IEEE Transactions on Broadcasting, vol. 48, no. 2, pp. 158-162, Jun. 2002.

[8] P. J. Edmonson, P. M. Smith, C. K. Campbell, "SAW injection locked oscillators: dynamic behaviour and application to neural networks". In: 1993 IEEE Ultrasonics Symposium, 1993, Baltimore. Proceedings... Maryland, USA: IEEE, 1993, vol. 1, pp. 131-135. 